\section{P-39 INTEGRATED CARE SYSTEM (ICS) APPROACH TO DIGITAL TRANSFORMATION OF ADVANCE CARE PLANNING}

Julia Neale, Sarah Onions, Graeme Still. Herefordshire and Worcestershire CCG, Worcester, UK

10.1136/spcare-2021-Hospice.60

Background Advance care plans (ACP) allow individuals to specify their wishes and preferences for future treatment and care. An effective ACP needs to be available to the patient and all relevant health and care professionals (Lund, Richardson, May, 2015). Currently there is no consistent approach to recording, updating and contemporary sharing of an individual's end-of-life care preferences, decisions and plans between health and care providers, patients and their family or carers in our Integrated Care System (ICS).

Aim Current ICS strategic priorities include developing personalised care planning approaches to improve shared decision making. Digital sharing of ACPs in real time, accessible to all health care providers, and to patients, carers and families via an online patient portal will support patients to share what really matters to them, reduce the need to re-tell their stories and achieve better care preferences.

Methods A key enabler was the developing system-wide digital shared care record programme. To deliver a digital ACP collaboration between patients, carers and health and care providers was facilitated by a focused review of current approaches to ACP, delivery of virtual workshops based on a co-production approach, and wide stakeholder involvement.

Results As part of a wider shared care record across the ICS, the digital ACP will deliver an accessible person-centred, real time record. Equality, diversity and inclusivity especially digital exclusion, have been addressed throughout the project. Synergies with the national 'What Matters conversation movement'(End of Life Care Think Tank, 2021) and the personalised care (NHS: Delivering universal personalised care) agenda have also been prioritised.

Outcome On completion of the technical build, user acceptance testing will be performed before implementation. The ACP digital document will then 'travel' with patients wherever their care takes place, whether at home, ambulance, hospital, care home or hospice. Real time updating ensures their plan will be contemporary, supporting 'What Matters' to individual patients to remain central to the care they receive.

\section{P-40 ONLINE SIMULATION TO EDUCATE THE WORKFORCE IN BREAKING BAD NEWS TO PEOPLE WITH LEARNING DISABILITIES}

Helen Needham, Kara Fereday. Birmingham City University, Birmingham, UK

\subsection{6/spcare-2021-Hospice.61}

Background Using actors in end of life scenario based simulation as a teaching method, allows for exploration of communication, emotional responses and authenticity in developing confidence and competence (MacLean, Kelly, Geddes, et al., 2017; Coleman \& McLaughlin, 2019). In response to the COVID-19 pandemic, this abstract's co-author initiated interactive, face-to-face online scenario based simulation across preregistration nursing at Birmingham City University (Warren et al, 2021). Evidence shows us that people with learning disabilities experience extensive health inequalities which impacts on mortality (Heslop, Blair, Fleming et al., 2013; National Institute for Health Research, 2020). In addition the quality of palliative care received by this group can contribute to poorer outcomes that are often avoidable (Hospice UK, 2021). However, using suitable methods of communication to impart information in a format that allows for deeper understanding opens up potential for greater quality of life and expectancy (Heslop, Blair, Fleming et al., 2013; National Institute for Health Research, 2020).

Aim To practice the skill of breaking bad news to people with learning disabilities, by using actors in simulated end-of-life patient scenarios.

Methods On MS Teams in small groups, with a facilitator, to engage all learners in an interactive, authentic and safe experience with an actor. Varied scenarios were used to demonstrate breaking bad news to a person with learning disabilities. A multipoint positive feedback approach was adopted.

Results Reflective evaluative feedback from students demonstrate a deeper confidence in breaking bad news. This included a perceived lessening of anxiety when involved in discussing breaking bad news in practice generally. Confirmation of online simulation as a valuable practical skills-based learning experience.

Conclusion This is now embedded in the future nurse curriculum and is applicable to a wider workforce approach including post-registration practitioners - both generalist and those working within learning disabilities and end-of-life care. This activity is being considered for inclusion in the co-authors' future PhD studies.

\section{P-41 ABSTRACT WITHDRAWN}

\section{P-42 EUPHEMISMS WHEN DESCRIBING DEATH AND DYING IN SPECIALIST PALLIATIVE CARE: AN OBSERVATIONAL SURVEY}

${ }^{1} J o n$ Choy, ${ }^{2}$ Andrew Thorns, ${ }^{2}$ Charlotte Brigden. 'Maidstone Hospital, Maidstone, UK 2Pilgrims Hospices in East Kent, Canterbury, UK

\subsection{6/spcare-2021-Hospice.62}

Background Euphemisms are frequently used to replace the words 'death' and 'dying' in the general population as well as within healthcare settings. Their use risks unclear communication leading to complaints in healthcare sectors ( Magnus, Fox, Elster, et al., 2015). The General Medical Council states: 'you must do your best to explain clinical issues in a way the person can understand'(General Medical Council. Treatment and care towards the end of life: working with the principles and decision-making models).

Encouraging people to openly talk about death and dying enables them to make informed decisions about their future health care and treatment (Lakasing, 2014). Research has shown that patients and caregivers prefer communication using direct language, avoiding euphemisms (Collins, McLachlan, \& Philip, 2018).

Aim To observe the use of the 'd-words' (death/dying/died) compared to euphemisms in a hospice multi-disciplinary team. 